\title{
Accidental Mercury Ingestion in a 2-Year Old: A Case Report
}

\section{Priya B* and Varshini C}

Department of Paediatrics, Indira Child Care, Chennai, India

*Corresponding Author: Priya B, Consultant, Department of Paediatrics, Indira Child Care, Chennai, India.
Received: July 29, 2020

Published: September 16, 2020

(C) All rights are reserved by Priya B and

Varshini C.

\section{Abstract}

Mercury exists in three forms- elemental (liquid mercury), inorganic mercury and organic mercury (methyl mercury). Elemental mercury or quick silver is the most commonly available form that is processed from an ore called cinnabar. Ingestion of elemental mercury can occur as an occupational or domestic hazard, mishandling or as an attempt to suicide. Although the oral ingestion of elemental mercury is unlikely to cause systemic toxicity due to its poor absorption by the gastrointestinal tract, it can cause adverse effects during any developmental period. Here we present a case of a 2-year old male child who accidentally ingested mercury from a household mercury thermometer. The child did not show any severe signs of toxicity or other systemic symptoms and was stable in the 24th and 48th hours following ingestion and after the 8th week follow-up. His blood parameters, vital signs, RR, HR, LFT and RFT were normal. X-ray abdomen showed radiopaque spots. The aim of this case report is to create awareness among Paediatricians, nurses and other health care workers to understand the systemic effects of mercury and prepare them to handle such cases in the medical practice. Mercury and its toxic effects may be taken as granted in the domestic; therefore, more awareness must be created in the patient population too, to stop the domestic use of mercury thermometers and encourage the use of infrared thermometers. This case report also presents an extensive review of mercury and effects of poisoning. Keywords: Liquid Mercury; Organic Mercury; Mercuric Salts

\section{Introduction}

Mercury is a heavy metal of known toxicity, that exists in several forms: organic, inorganic and elemental mercury. Inorganic mercury includes metallic mercury, mercury vapour $\left(\mathrm{Hg}^{0}\right)$, mercurous mercury $\left(\mathrm{Hg}^{2++}\right)$ and mercuric salts $\left(\mathrm{Hg}^{++}\right)$. Organic mercury are those bonded to a structure with carbon atoms like methyl, ethyl or phenyl groups [1]. The molecular behavior, pharmacokinetics and clinical significance differs with their chemical structure and the route of absorption and presents differently for the various forms of mercury [2].

Elemental mercury vapor is inhaled and easily absorbed through the mucus membrane of lungs and is quickly oxidized to the other forms while methyl/organic mercury is absorbed through the gut. Elemental mercury crosses the blood brain barrier more efficiently but methyl mercury can be progressively demethylated to elemental mercury on entering the brain. Mercury salts are on the other hand insoluble and poorly absorbed and are relatively stable [3]. Inhaled mercury primarily targets the brain and mercuric salts affect the kidney and GIT lining, while organic mercury is distributed throughout the body. The toxicity differs with the form, dose and rate of mercury exposure [4]. Acute large exposures of elemental mercury vapor can cause pneumonitis and can be fatal. Low grade chronic exposure can cause mild systemic symptoms over the time which includes fatigue, anorexia, weight loss and gastrointestinal stress, also called as micromercurialism. High grade chronic exposure can cause tremors, erethism, gingivitis and sialorrhoea [5].

Elemental mercury is easily available and can cause occupational and domestic hazards. It has high vapor pressure and is less 
toxic than inorganic and organic bound mercury in room temperature [6]. It is a shiny silver coloured liquid that can be used in thermometers, sphygmomanometers, batteries and barometers. They can also be used in silver and gold processing [7]. Elemental mercury evaporates at room temperature and intoxication is usually via inhalational route. Only less than $0.1 \%$ is absorbed through GIT following oral ingestion [8].

The accidental oral intake of elemental mercury in children is rare and here we present a case of a 2-year old male child who reported to our emergency department with ingested mercury due to accidental breakage of thermometer and still remained asymptomatic.

\section{Case Report}

A 2-year old male child was brought by the mother to the emergency department with the complaint of accidental breakage of mercury thermometer in the child's mouth and probable ingestion of liquid mercury1.5 hours back. The child was febrile for the past 2 days and the mother had used the thermometer to record the temperature. The mother was concerned and feared that the child might have ingested the mercury. He had also had 1-2 episodes of vomiting earlier that day but no $\mathrm{H} / \mathrm{O}$ vomiting after this incident. There were no H/O loose motions, irritability, drowsiness, LOC (loss of consciousness), seizures or coughing. There were also no complaints of pain in the mouth, breathing difficulty or abdominal pain. No oral intake was given following this accident.

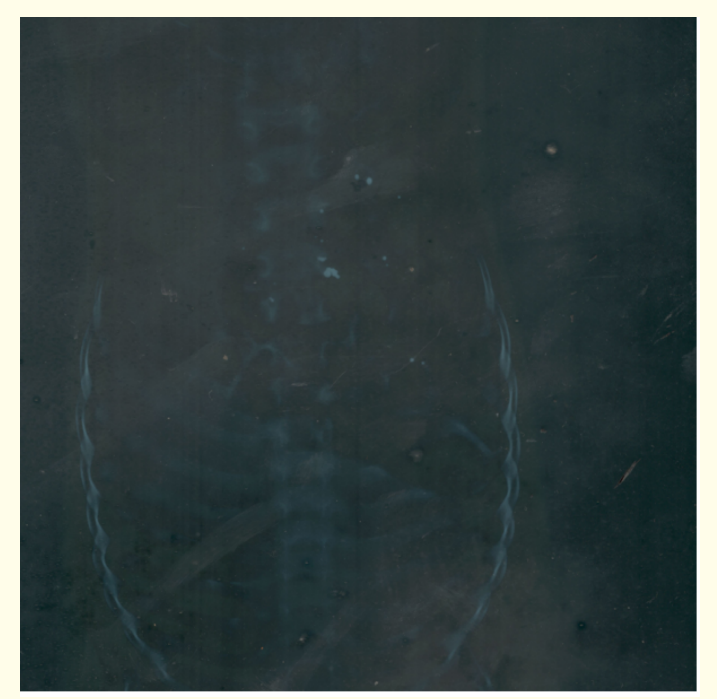

Figure 1: Abdominal radiograph showing multiple round densities scattered in gastrointestinal tract.
On examination, the child was febrile $\left(100^{\circ} \mathrm{F}\right)$, playing, conscious, oriented and answering appropriately. A small bruise near right corner of mouth was observed which could be due to injury from the broken thermometer. No anemia, palloror or cyanosis was observed. His respiratory rate was- $32 / \mathrm{m}$, heart rate $124 \mathrm{bpm}$, BP- 92/60 $\mathrm{mm} \mathrm{Hg}$ and $\mathrm{PaO}_{2}=99 \%$ in room temperature and air. Upon examination of the respiratory system, bilateral equal air entry was observed. No creps /wheeze was heard. CVS examination showed $\mathrm{S}_{1}, \mathrm{~S}_{2}+$; Pulses were equal in all 4 limbs and the child was not tachypnoeic. Abdomen was soft to palpation and no tenderness ororganomegaly was observed.

The child was stable, conscious with normal vitals at the time of examination. Oral examination revealed a small $1 \mathrm{~cm}$ linear cut near the right corner of the mouth. No bruising, ulceration or discoloration was observed intra-orally. Throat, CNS and other systemic examinations were normal. $\mathrm{X}$ ray abdomen and chest was done which showed a few hyperdensespots in stomach area. The child was admitted in the hospital and kept for observation since the amount of mercury ingested was not clear.

His CBC and peripheral smear were normal. Liver and renal function tests (LFT, RFT) were also normal. Serum mercury level was $<3 \mu$ g. He had an uneventful stay in hospital and was discharged after 24 hours. Follow-up X-ray was done 24 and 48 hours later which showed residual spots in the lower GIT. Repeat X-ray was done after 8 weeks and was found normal. The radiograph showed complete clearance of mercury from the GIT. The child remained stable in the follow up.

\section{Discussion}

According to the WHO, the most common human exposure of mercury is by outgassing of mercury from dental amalgam, occupational exposure like coal burning and mining, and from ingestion of contaminated sea food [9]. Atmospheric exposure is usually through volcanic activity and outgassing from rock. Atmospheric mercury settles in water and is converted into organic (methyl and ethyl) mercury by microorganisms and is eventually consumed by small creatures which in turn are ingested by larger fish like tuna, swordfish or shark. These fish may have considerable amount of mercury in their tissues and their consumption may lead to mercury poisoning in humans in the long term [10]. Many lakes in the United States have been closed to fishing due to mercury burden.

The chief route of mercury exposure is the respiratory route by inhalation of mercuric vapours, especially from dental amalgams. 
Dentists and staff working in a dental set up are high risk categories for mercury toxicity. Mercury outgassing from a dental amalgam filling occurs at a rate of 2- $28 \mu$ g per facet surface per day and the absorption rate is about $80 \%$ according to a report by WHO and Berlglund., et al. [11,12]. Acute exposure to mercury vapour can

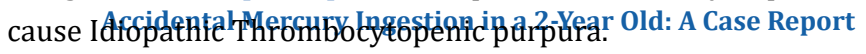

The chief pathway of elemental mercury exposure in children are from dental amalgam fillings (risk of ingestion of broken fillings), take home exposure from adults who are occupationally exposed and accidental ingestion from thermometers, manometers, batteries and fluorescent light bulbs. Children who are involved in gold extraction process and mining are also at risk of mercury exposure [11]. They are also at risk of exposure from certain skin lightening cosmetics, interior latex paints and other traditional forms of ayurvedic treatments where mercuric candles are burnt. They are also exposed to elemental mercury at school laboratories where they are attracted by its shiny silver color and tendency to form beads [9]. The exposure of organic mercury in children is from consumption of methyl mercury contaminated sea-food.

The main route of liquid mercury exposure in children is by ingestion but it is not that poisonous due to the limited absorption from the GI tract. The reason behind this low GI absorption is that mercury is first converted into vapour and this vapour binds quickly to the sulfhydryl groups in the GI tract. This in turn has a low impact on human health when compared to the ingestion of organic or inorganic mercury [13]. Methylmercury can be excreted into breast milk and can be ingested by sucklers. Therefore, pregnant women must avoid frequent intake of shark, King mackerel, swordfish and tuna as they contain large amounts of mercury.

$70-85 \%$ of the mercury vapours are inhaled by the lungs and absorbed into the blood stream. The mercury vapours migrate from the pharynx into the brain through the olfactory neurons. Animal studies show that methylmercury vapours are rapidly and completely absorbed into the blood stream and is the most dangerous route [7]. Elemental and organic mercury can cross the placenta and enter the fetus as the fetus is incapable of excreting mercury. Transplacental route of mercury absorption was first observed in Minamata Bay in Japan due to overconsumption of seafood [1]. Phenyl mercury can also be transdermally absorbed from contaminated diapers in infants.

The symptoms of mercury intoxication can be acute or chronic. Symptoms vary depending on the dose and length of exposure. The most characteristic acute symptoms include the airway which are cough and dyspnoea and pneumonitis. Fever, headaches and CNS disturbances like tremors, ataxia, disturbance in motor coordination, dysdiadochokinesia, sensation difficulties, abnormal reflexes and loss of consciousness may be present [14]. Oral symptoms can show as marginal gingivitis, stomatitis and sialorrhoea. Mercurial erethism is a combination of excitability, memory loss, insomnia and extreme shyness. Proteinuria and damages to lining cells of kidney may be observed. Chronic exposure can cause Acrodynia (Pink's disease) with painful and swollen extremities, pinkish discolouration, peeling and erythema [15]. They are also proven to cause teratogenicity, CVS toxicity (tachycardia, hypertension, heart rate variability). High amounts of exposure to methyl mercury can also be carcinogenic and immunotoxic. They can also cause still birth and reproductive toxicity [16].

A lack of correlation between the symptoms and amount of exposure is observed in many cases and there is a risk of misdiagnosis. Diagnosis of mercury toxicity is mainly by eliciting a proper medical and environmental history. A complete physical examination, blood and urine analysis must be done. Chest and abdominal $\mathrm{X}$-rays may assist in identifying ingested mercury. Management of mercury poisoning depends on the amount of ingested or inhaled mercury, its absorption into the blood stream and the length of exposure, severity of symptoms, patient susceptibility and expertise of the treating doctor. In the presence of high blood and urine mercury levels, respiratory distress with acrodynia, chelation treatment with NAC (N-Acetylcysteine) must be started. Other chelating drugs used are dimercaprol (BAL), dimercaprosuccinic acid (DMSA), 2,3-Dimercapropropane-1-sulphonate (DMPS) and British Anti Lewisite (BAL) [17]. Proper follow-up and monitoring of the patient is essential.

Children are likely to be exposed to different routes and forms of mercury. In our case, the child had ingested $<3 \mu$ g of elemental mercury with no toxic signs. However, the effects may be different in other cases and children. Therefore, this is an attempt to review the current approach to elemental mercury poisoning and create an awareness among the medical staff with the hope of further contributions in this area.

\section{Corollary}

Phasing out of mercury thermometer and encouraging the use of infrared thermometer. 


\section{Conclusion}

The protection of children's health by the family and community must be motivated. Children are often exposed to elemental mercury due to inappropriate handling and clean up. Health education to the parents, paediatricians and hospital staff are needed as primary prevention. Policy initiatives must be implemented to protect children.

\section{Bibliography}

1. World Health Organization. Exposure to mercury: A major public health concern. WHO, Public Health and Environment Geneva (2007).

2. Clarkson TW., et al. "The toxicology of mercury-current exposures and clinical manifestations". The New England Journal of Medicine349 (2003): 1731-1737.

3. Bernhoft RA. "Mercury toxicity and treatment: a review of the literature". Journal of Environmental and Public Health (2012): 460508.

4. M Berlin., et al. "'Mercury". Handbook on the Toxicology of Metals, G. F. Nordberg, B. A. Fowler, M. Nordberg, and L. T. Friberg, Eds., chapter 33, Elsevier, New York, NY, USA, 3rd edition (2007).

5. SB Skerfving and JF Copplestone. "Poisoning caused by the consumption of organomercury dressed seed in Iraq". Bulletin of the World Health Organization 54.1 (1976): 101-112.

6. Boyd AS., et al. "Mercury exposure and cutaneous disease". Journal of the American Academy of Dermatology 43 (2000): 81-90.

7. McKay CA., et al. "Mercury". In: Erickson TB, Baum CR, Ling LJ, eds, Pediatric Toxicology: Diagnosis and Management of the Poisoned Child. NewYork, PA: The Mc- Graw-Hill Companies (2005): 468-475.

8. Kolkhorst KS and Kulkarni PM. "Accidental metallic mercury in- gestion". The Journal of the American Osteopathic Association 112 (2012): 381.

9. World Health Organization. "Inorganic mercury: environmental health criteria 118". International Programme on Chemical Safety, World Health Organization, Geneva, Switzerland (1991).
10. HM Boylan., et al. "A new method to assess mercury emissions: a study of three coal-fired electric-generating power station configurations". Journal of the Air and Waste Management Association 53.11 (2003): 1318-1325.

11. M Richardson. The Safety of Dental Amalgam, ISBN 0-66224873-2, Minister of Health, Canada (1996).

12. A Berglund., et al. "Determination of the rate of release of intra-oral mercury vapor from amalgam". Journal of Dental Research 67.9 (1988): 1235-1242.

13. Mahajan PV. "Heavy Metal Intoxication Mercury". In: Kliegman RM, Geme JW, Schor NF, Behrman RE, eds, Nelson Textbook of Pediatrics. 19th ed. Philadelphia PA: Elsevier Saunders (2011): 2448.

14. JG Schwartz., et al. "Toxicity of a family from vacuumed mercury". American Journal of Emergency Medicine 10.3 (1992): 258-261.

15. Agency for Toxic Substances and Disease Registry - ATSDR. Public Health Service. Toxicological Profile for Mercury (1999).

16. Tchounwou PB., et al. "Environmental exposure to mercury and its toxicopathologic implications for public health". Environment Toxicology 18 (2003): 149-175.

17. HV Aposhian. "DMSA and DMPS-water soluble antidotes for heavy metal poisoning". Annual Review of Pharmacology and Toxicology 23 (1983): 193-215.

\section{Assets from publication with us}

- Prompt Acknowledgement after receiving the article

- Thorough Double blinded peer review

- Rapid Publication

- Issue of Publication Certificate

- High visibility of your Published work

Website: www.actascientific.com/

Submit Article: www.actascientific.com/submission.php

Email us: editor@actascientific.com

Contact us: +919182824667 